\title{
WEAKLY HOMOGENEOUS MODELS ${ }^{1}$
}

\begin{abstract}
ANAND PILLAY
ABSTRACT. I consider some notions of weak homogeneity, which generalise $\omega$-homogeneity. I first analyse a specific such notion, called almost homogeneity, in the context of $\omega$-stable theories. (Almost homogeneity is just like $\omega$-homogeneity, but using strong types in place of types.) Then in a more general context, I prove for weakly homogeneous countable models some classification results which are known for $\omega$-homogeneous countable models, in particular the result that the isomorphism type of such a model is determined by the types which it realises.
\end{abstract}

0. Introduction and preliminaries. We are concerned with models (mainly countable) and theories, in countable languages. The notion of homogeneity is very important for classifying countable models. Namely, if $M$ and $N$ are countable models, $N$ is $\omega$-homogeneous and all types realised in $M$ are realised in $N$, then $M$ is elementarily embeddable in $N$. Also if $M$ and $N$ are both $\omega$-homogeneous and realise the same types, then they are isomorphic. This shows that if all countable models of the complete theory $T$ are $\omega$-homogeneous then $n(T)$, the number of countable models of $T$, is $1, \boldsymbol{\aleph}_{0}$ or $2^{\aleph_{0}}$. It turns out that the same is true using weaker notions than homogeneity. To say that a countable model $M$ is $\omega$-homogeneous means that for each type $p(\bar{x}) \in S(\operatorname{Th}(M))$, all members of $p^{M}$ are conjugate via automorphisms of $M$. Part of the content of weak homogeneity says that for each such $p, p^{M}$ is divided into a finite number of conjugacy classes by automorphisms of $M$. There can be other ramifications of the notion depending for example on whether or not the conjugacy relation above is "definable", or on whether a "back-and-forth" definition of weak homogeneity is used. A model $M$ will be called "almost homogeneous" if whenever $\bar{a}, \bar{b}, c \in M$ and $\operatorname{stp}(\bar{a})=\operatorname{stp}(\bar{b})$, then there is $d \in M$ such that $\operatorname{stp}\left(\bar{a}^{\wedge} c\right)=\operatorname{stp}\left(\bar{b}^{\wedge} d\right)$. (stp refers to strong type.) If the theory $T$ (whose models we are considering) is $\omega$-stable, or even stable with all type having finite multiplicity, then almost homogeneity will be a weak notion of homogeneity as described above. Almost homogeneity is particularly interesting as it has been shown (in [1]) that for a large class of $\omega$-stable theories $T$ (which includes the nonmultidimensional theories), all models of $T$ are almost homogeneous. In $\S 1, I$ analyse almost homogeneous models of $\omega$-stable theories. In $\$ 2$, I present a general notion of weak homogeneity, and I strengthen to such models the classification results for homogeneous models mentioned above.

Received by the editors March 9, 1981 and, in revised form, September 14, 1981. 1980 Mathematics Subject Classification. Primary 03C15, 03C50; Secondary 03C45.

$K e y$ words and phrases. Strong type, $\omega$-stable, almost homogeneous, $E$-homogeneous.

'The work presented here was done while the author was visiting the University of Paris VII in the autumn of 1980, as part of an ATP Internationale project, funded by the C.N.R.S. whom I thank. 
I thank the referee for his suggestions concerning the presentation of the material, as well as for shortening the proof of Proposition 20, and simplifying Examples 13 and 14.

I follow the usual convention of working in a big saturated model $\bar{M}$ of whichever theory we are talking about. Thus all models will be considered as elementary submodels of $\bar{M}$. By tuples, I shall mean finite tuples of elements of $\bar{M}$, denoted $\bar{a}, \bar{b}$, etc. We recall the following definitions from [3].

Definition 1. Let $A$ be a subset (of $\bar{M}$ ). A formula $\varphi(\bar{x}, \bar{y}$ ) is said to be a finite equivalence relation over $A$, if $\varphi$ has parameters from $A$ only, $l(\bar{x})=l(\bar{y})=n$ for some $n<\omega$, and $\varphi$ defines an equivalence relation with finitely many classes on the set of $n$-tuples from $\bar{M}$. The set of all finite equivalence relations over $A$ is denoted by $\operatorname{FE}(A)$.

Definition 2. Let $\bar{a}, \bar{b}$ be tuples of the same length $n$ say, and $A$ a set of parameters. We say $\operatorname{stp}(\bar{a} / A)=\operatorname{stp}(\bar{b} / A)$ if for all $E(\bar{x}, \bar{y}) \in \operatorname{FE}(A)$ with $l(\bar{x})=$ $l(\bar{a}), \vDash E(\bar{a}, \bar{b})$. If $A=\varnothing$, we omit it.

Note 3. $\operatorname{stp}(\bar{a} / A)=\operatorname{stp}(\bar{b} / A)$ implies $\operatorname{tp}(\bar{a} / A)=\operatorname{tp}(\bar{b} / A)$.

The reader can consult [2] or [3] for notions from stability theory, especially that of forking. I will state explicitly some of the pertinent facts.

FACT 4 . Let $T$ be stable, $\bar{a}, \bar{b}$ be tuples of the same length and $A$ be a set. Then the following are equivalent:

(i) $\operatorname{stp}(\bar{a} / A)=\operatorname{stp}(\bar{b} / A)$,

(ii) for all $B \supset A$, if $\operatorname{tp}(\bar{a} / B)$ does not fork over $A$ and $\operatorname{tp}(\bar{b} / B)$ does not fork over $A$ then $\operatorname{tp}(\bar{a} / B)=\operatorname{tp}(\bar{b} / B)$,

(iii) for some model $M \supset A, \operatorname{tp}(\bar{a} / M)=\operatorname{tp}(\bar{b} / M)$.

If $T$ is stable and $p \in S(A)$, then the multiplicity of $p$ is the maximum number of nonforking extensions over any $B \supset A$ (which equals the number of nonforking extensions of $p$ over some model $M \supset A$ ). A consequence of Fact 4 is:

FACT 5. Let $T$ be stable. Let $p(\bar{x}) \in S(A)$ have finite multiplicity. Then there is $E(\bar{x}, \bar{y}) \in \operatorname{FE}(A)$ such that $p(\bar{x}) \cup p(\bar{y}) \cup\{E(\bar{x}, \bar{y})\} \vdash E^{\prime}(\bar{x}, \bar{y})$ for all $E^{\prime} \in \operatorname{FE}(A)$. Another way of saying this is $p(\bar{x})+$ ' $E$-class of $x$ ' $+\operatorname{stp}(\bar{a} / A)$.

FACT 6. Let $T$ be stable and $p \in S(A)$. Then the multiplicity of $p$ is either finite or $2^{\kappa_{0}}$.

A consequence of Fact 6 is

FACT 7. $T$ is $\omega$-stable if and only if (i) $T$ is superstable ( $T$ is stable and for any $B$ and $p \in S(B)$ there is finite $A \subset B$ such that $p$ does not fork over $A$ ), (ii) for all $A$ and $p \in S(A), p$ has finite multiplicity, and (iii) $S(T)$, the set of pure types of $T$, is countable.

\section{Almost homogeneous models of $\omega$-stable theories.}

Definition 8. The model $M$ will be called almost homogeneous if for all $\bar{a}, \bar{b}, c \in$ $M, \operatorname{stp}(\bar{a})=\operatorname{stp}(\bar{b})$ implies that there is $d \in M$ such that $\operatorname{stp}\left(\bar{a}^{\wedge} c\right)=\operatorname{stp}\left(\bar{b}^{\wedge} d\right)$.

DEFINITION 9. An automorphism $f$ of a model $M$ is said to be a strong automorphism if for all tuples $\bar{a}$ in $M \operatorname{stp}(\bar{a})=\operatorname{stp}(f(\bar{a}))$. 
The obvious back and forth argument shows that a countable model $M$ is almost homogeneous iff whenever $\bar{a}, \bar{b} \in M$ and $\operatorname{stp}(\bar{a})=\operatorname{stp}(\bar{b})$ there is a strong automorphism $f$ of $M$ such that $f(\bar{a})=\bar{b}$. If $T$ is stable and all types have finite multiplicity, then by Fact 5 , if $\bar{a}, \bar{b} \in M$, and $\operatorname{tp}(\bar{a})=\operatorname{tp}(\bar{b})$, then there is $E \in \mathrm{FE}(\varnothing)$ such that if $M \vDash E(\bar{a}, \bar{b})$ then $\operatorname{stp}(\bar{a})=\operatorname{stp}(\bar{b})$ (where $E$ depends only on $\operatorname{tp}(\bar{a}))$. Thus in this case, if $M$ is countable and almost homogeneous, then for any $p(\bar{x})$ realised in $M$, $p^{M}$ is divided into a finite number of orbits (or conjugacy classes) by automorphisms of $M$.

It is not clear, even if $T$ is stable and all types have finite multiplicity, that if $M$ is $\omega$-homogeneous then $M$ is almost homogeneous. (If $M$ is $\omega$-homogeneous, $\bar{a}, \bar{b}, c \in M$ and $\operatorname{stp}(\bar{a})=\operatorname{stp}(\bar{b})$, then there will clearly be $d \in M$ such that $\operatorname{tp}\left(\bar{a}^{\wedge} c\right)=\operatorname{tp}\left(\bar{b}^{\wedge} d\right)$, but there will not obviously be $d \in M$ such that $\operatorname{stp}\left(\bar{a}^{\wedge} c\right)=\operatorname{stp}\left(\bar{b}^{\wedge} d\right)$.) However if $T$ is $\omega$-stable, this will be the case:

Proposition 10. Let $M$ be a countable model of the $\omega$-stable theory $T$. Then the following are equivalent:

(i) $M$ is almost homogeneous,

(ii) if $\bar{a}, \bar{b} \in M$ and $\operatorname{stp}(\bar{a})=\operatorname{stp}(\bar{b})$, then there is a strong automorphism $f$ of $M$ such that $f(\bar{a})=\bar{b}$,

(iii) if $\bar{a}, \bar{b} \in M$ and $\operatorname{stp}(\bar{a})=\operatorname{stp}(\bar{b})$ then there is an automorphism $f$ of $M$ such that $f(\bar{a})=\bar{b}$,

(iv) if $\bar{a}, \bar{b}, c \in M$ and $\operatorname{stp}(\bar{a})=\operatorname{stp}(\bar{b})$ then there is $d \in M$ such that $\operatorname{tp}\left(\bar{a}^{\wedge} c\right)=$ $\operatorname{tp}\left(\bar{b}^{\wedge} d\right)$.

Proof. (i) $\Rightarrow$ (ii) follows, as mentioned above, by the obvious back and forth.

(ii) $\Rightarrow$ (iii) and (iii) $\Rightarrow$ (iv) are trivial.

(iv) $\Rightarrow$ (i). Let $\bar{a}, \bar{b}$ and $c$ be in $M$ such that $\operatorname{stp}(\bar{a})=\operatorname{stp}(\bar{b})$. We seek $d$ in $M$ such that $\operatorname{stp}\left(\bar{a}^{\wedge} c\right)=\operatorname{stp}\left(\bar{b}^{\wedge} d\right)$. Let $M_{1}$ be an elementary substructure of $M$ which contains $\bar{a}$ and is prime over $\bar{a}$. ( $M_{1}$ exists by Fact 7(iii).) Now by Fact 5 and Fact 7 (i) and (ii), there is a finite tuple $\bar{a}^{\prime}$ in $M_{1}$ such that $\operatorname{tp}\left(c / M_{1}\right)$ does not fork over $\bar{a}^{\wedge} \bar{a}^{\prime}$ and $\operatorname{tp}\left(c / \bar{a}^{\wedge} \bar{a}^{\prime}\right)+\operatorname{stp}\left(c / \bar{a}^{\wedge} \bar{a}^{\prime}\right)$.

I will now show that there is $\bar{b}^{\prime}$ in $M$ such that $\operatorname{stp}\left(\bar{a}^{\wedge} \bar{a}^{\prime}\right)=\operatorname{stp}\left(\bar{b}^{\wedge} \bar{b}^{\prime}\right)$. As $\operatorname{stp}(\bar{a})=\operatorname{stp}(\bar{b})$, there is $\bar{e}$ in the big model $(\bar{M})$, such that $\operatorname{stp}\left(\bar{a}^{\wedge} \bar{a}^{\prime}\right)=\operatorname{stp}\left(\bar{b}^{\wedge} \bar{e}\right)$. Let $p$ be $\operatorname{tp}\left(\bar{a}^{\wedge} \bar{a}^{\prime}\right)$, and let $E$ be the finite equivalence relation (over $\varnothing$ ) corresponding to $p$ as given by Fact 5. (Remember $p$ has finite multiplicity.) Let $\alpha(\bar{a}, \bar{x})$ be a formula which isolates $\operatorname{tp}\left(\bar{a}^{\prime} / \bar{a}\right)$. Thus clearly $\approx \alpha(\bar{b}, \bar{e}) \wedge E\left(\bar{a}^{\wedge} \bar{a}^{\prime}, \bar{b}^{\wedge} \bar{e}\right)$. Now pick $\bar{b}^{\prime}$ in $M$ such that $M=\alpha\left(\bar{b}, \bar{b}^{\prime}\right) \wedge E\left(\bar{a}^{\wedge} \bar{a}^{\prime}, \bar{b}^{\wedge} \bar{b}^{\prime}\right)$. As $\operatorname{tp}(\bar{a})=\operatorname{tp}(\bar{b}), \alpha(\bar{b}, \bar{x})$ isolates $\operatorname{tp}\left(\bar{b}^{\prime} / \bar{b}\right)$ and moreover $\operatorname{tp}\left(\bar{b}^{\wedge} \bar{b}^{\prime}\right)=\operatorname{tp}\left(\bar{a}^{\wedge} \bar{a}^{\prime}\right)=p$. Thus by choice of $E$ and Fact 5 , it follows that $\operatorname{stp}\left(\bar{a}^{\wedge} \bar{a}^{\prime}\right)=\operatorname{stp}\left(\bar{b}^{\wedge} \bar{b}^{\prime}\right)$, as required.

Now by (iv) there is $d$ in $M$ such that $\operatorname{tp}\left(\bar{a}^{\wedge} \bar{a}^{\prime} \wedge c\right)=\operatorname{tp}\left(\bar{b}^{\wedge} \bar{b}^{\wedge} d\right)$. I assert that in fact $\operatorname{stp}\left(\bar{a}^{\wedge} \bar{a}^{\prime \wedge} c\right)=\operatorname{stp}\left(\bar{b}^{\wedge}{\overline{b^{\prime}}}^{\wedge} d\right)$.

First note that $\operatorname{tp}\left(d / \bar{b}^{\wedge} \bar{b}^{\prime}\right)+\operatorname{stp}\left(d / \bar{b}^{\wedge} \bar{b}^{\prime}\right)$ (by (*) and the fact that $\bar{a}^{\wedge} \bar{a}^{\wedge} c$ and $\bar{b}^{\wedge}{\overline{b^{\prime}}}^{\wedge} d$ have the same type). $\quad(* *)$ 
Let us suppose that $\operatorname{stp}\left(\bar{a}^{\wedge} \bar{a}^{\wedge} c\right) \neq \operatorname{stp}\left(\bar{b}^{\wedge} \bar{b}^{\wedge} \wedge d\right)$ and look for a contradiction. As $\operatorname{stp}\left(\bar{a}^{\wedge} \bar{a}^{\prime}\right)=\operatorname{stp}\left(\bar{b}^{\wedge} \bar{b}^{\prime}\right)$, there is $d^{\prime}$ in the big model such that $\operatorname{stp}\left(\bar{a}^{\wedge} \bar{a}^{\prime} \wedge c\right)=$ $\operatorname{stp}\left(\bar{b}^{\wedge} \bar{b}^{\prime} \wedge d^{\prime}\right)$. Thus $\operatorname{stp}\left(\bar{b}^{\wedge}{\overline{b^{\prime}}}^{\wedge} d^{\prime}\right) \neq \operatorname{stp}\left(\bar{b}^{\wedge} \bar{b}^{\prime} \wedge d\right)$. So there is $E \in \operatorname{FE}(\varnothing)$ such that $\vDash \neg E\left(\bar{b}^{\wedge} \bar{b}^{\prime} \wedge d^{\prime}, \bar{b}^{\wedge} \bar{b}^{\prime} \wedge d\right)$. Let us define $E^{\prime}\left(x_{1}, x_{2}\right)$ by

$$
E^{\prime}\left(x_{1}, x_{2}\right) \text { if }=E\left(\bar{b}^{\wedge} \bar{b}^{\prime} \wedge x_{1}, \bar{b}^{\wedge} \bar{b}^{\wedge} \wedge x_{2}\right) \text {. }
$$

Then clearly $E^{\prime} \in \operatorname{FE}\left(\bar{b}^{\wedge} \bar{b}^{\prime}\right)$, and moreover $\vDash_{\neg} E^{\prime}\left(d^{\prime}, d\right)$. But it is clear that $\operatorname{tp}\left(d^{\prime} / \bar{b}^{\wedge} \bar{b}^{\prime}\right)=\operatorname{tp}\left(d / \bar{b}^{\wedge} \bar{b}^{\prime}\right)$, and thus we contradict $(* *)$. Thus we have shown that $\operatorname{stp}\left(\bar{a}^{\wedge} \bar{a}^{\prime \wedge} c\right)=\operatorname{stp}\left(\bar{b}^{\wedge} \bar{b}^{\prime} \wedge d\right)$ whereby clearly $\operatorname{stp}\left(\bar{a}^{\wedge} c\right)=\operatorname{stp}\left(\bar{b}^{\wedge} d\right)$.

REMARK 11. If $M$ is an uncountable model of an $\omega$-stable theory, then (i) and (iv) of Proposition 10 are still equivalent, by the same proof as above.

COROLlaRY 12. Let $M$ be an $\omega$-homogeneous model of an $\omega$-stable theory. Then $M$ is almost homogeneous.

We conjecture that Corollary 12 is not true if " $\omega$-stable" is replaced by "stable and all types have finite multiplicity".

I now give some examples and counterexamples.

EXAMPLE 13. An almost homogeneous model of an $\omega$-stable theory, which is not $\omega$-homogeneous.

Let $L$ be a language with binary relation symbol $R$, and let $L^{\prime}$ be $L \cup\{E\}$ where $E$ is also a binary relation symbol. Let $M$ be the $L$-structure $(Z, R)$ where $R(x, y)$ holds iff $x=n$ and $y=n+1$ for some $n \in \mathbf{Z}$. For any $\kappa$ let $M^{(\kappa)}$ denote the disjoint union of $\kappa$ copies of $M$. Clearly $M^{(\kappa)} \equiv M$. Now let $N$ be the $L^{\prime}$-structure in which $E$ is an equivalence relation with two classes, the $L$-reducts of which are $M$ and $M^{(2)}$. $\operatorname{Th}(N)$ is $\omega$-stable and $N$ is almost homogeneous. But $N$ is not homogeneous. For let $a$ be in one class and $b$ be in the other. Then $\operatorname{tp}(a)=\operatorname{tp}(b)$, but there is no automorphism of $N$ taking $a$ to $b$ for this would induce an isomorphism between $M$ and $M^{(2)}$.

EXAMPLE 14. A countable model $N$ of an $\omega$-stable theory, with elements $a_{0}$ and $a_{1}$ such that $\operatorname{stp}\left(a_{0}\right)=\operatorname{stp}\left(a_{1}\right)$, there is an automorphism of $N$ mapping $a_{0}$ to $a_{1}$, but there is no such strong automorphism. This example shows that Proposition 10 cannot be proved "locally".

Let $L$ and $M$ be as in the previous example, and now let $L^{\prime}=L \cup$ $\{P, Q, R, \alpha, \beta, E\}$, where these are all predicate symbols, $P, Q$ and $R$ being unary, $\alpha$ binary, $\beta$ ternary and $E$ 4-ary. (There will be some redundancy here, but it makes notation simpler.) $N$ will be the following $L^{\prime}$-structure. $P, Q$ and $R$ will partition $N$. $P^{N}=\left\{a_{i}: i<\omega\right\}$, and $Q^{N}=\left\{b_{i}^{k}: i<\omega, k<2\right\}$.

$\alpha(x, y)$ will hold iff there are $i<\omega, k<2$ such that $x=a_{i}$ and $y=b_{i}^{k}$.

$\beta(x, y, z)$ implies that $\alpha(x, y)$ and $R(z)$. If $R z$ then there are $x$ and $y$ such that $\beta(x, y, z)$. If $\left(x_{1}, y_{1}\right) \neq\left(x_{2}, y_{2}\right)$, then $\left\{z: \beta\left(x_{1}, y_{1}, z\right)\right\}$ and $\left\{z: \beta\left(x_{2}, y_{2}, z\right)\right\}$ are disjoint. Let us denote $\{z: \beta(x, y, z)\}$ by $\beta[x, y]$. Then $\beta\left[a_{0}, b_{0}^{0}\right] \uparrow L$ is $M$ and $\beta\left[a_{1}, b_{1}^{1}\right] \uparrow L$ is $M^{(2)}$. For all other $(x, y)$ such that $\alpha(x, y), \beta[x, y] \uparrow L$ is $M$. Finally $E\left(x_{1}, y_{1}, x_{2}, y_{2}\right)$ holds iff there are $i, j<\omega$, and $k<2$ such that $x_{1}=a_{i}, y_{1}=b_{i}^{k}$, $x_{2}=a_{j}$ and $y_{2}=b_{j}^{k}$. 
Now $\operatorname{Th}(N)$ is $\omega$-stable. Also $a_{0}$ and $a_{1}$ have the same strong type in $N$, and moreover there is an automorphism of $N$ taking $a_{0}$ to $a_{1}$. However there is no such strong automorphism. For suppose $f$ were such. Then $f$ would have to take $b_{0}^{0}$ to $b_{1}^{0}$. ( $f$ could not take $b_{0}^{0}$ to $b_{1}^{1}$, for because of $E, \operatorname{stp}\left(a_{0}, b_{0}^{0}\right) \neq \operatorname{stp}\left(a_{1}, b_{1}^{1}\right)$ ). But then $f$ would have to map $\beta\left[a_{0}, b_{0}^{0}\right]$ isomorphically onto $\beta\left[a_{1}, b_{1}^{0}\right]$, which is impossible, as the $L$-reducts of these are $M$ and $M^{(2)}$ respectively.

2. Classifying weakly homogeneous models. I first present a general context, motivated by suggestions of the referee.

Let $T$ be a (complete) theory. $E$ will denote an assignment, to each complete type $p(\bar{x})$ of $T$ and countable model $M$ of $T$, of an equivalence relation, denoted $E_{p}^{M}$, on $p^{M}$ the set of realisations of $p$ in $M$. Given such an assignment $E$, if $M$ is a (countable) model and $\bar{a}, \bar{b} \in M$, I will write $\bar{a} \sim_{M} \bar{b}$ to mean $\operatorname{tp}(\bar{a})=\operatorname{tp}(\bar{b})$ and $E_{\mathrm{tp}(\bar{a})}^{M}(\bar{a}, \bar{b})$. I give two properties which $E$ might satisfy:

(I) ${ }_{M}$ For all $p \in S(T), E_{p}^{M}$ has only a finite number of classes.

(II) Suppose that $M \prec N$ and $\bar{a}, \bar{b} \in M$. Then $\bar{a} \sim_{M} \bar{b}$ iff $\bar{a} \sim_{N} \bar{b}$.

LEMMA 15. Suppose that the assignment $E$ satisfies (II). Then it also satisfies

(III) For any $M$ and $\bar{a}, \bar{b} \in M$, the fact that $\bar{a} \sim_{M} \bar{b}$ depends only on $\operatorname{tp}\left(\bar{a}^{\wedge} \bar{b}\right)($ and not even on $M$ ).

Proof. Trivial, using the fact that if $\bar{a}, \bar{b} \in M, \bar{c}, \bar{d} \in N$, and $\operatorname{tp}\left(\bar{a}^{\wedge} \bar{b}\right)=\operatorname{tp}\left(\bar{c}^{\wedge} \bar{d}\right)$ then there are elementary embeddings of $M$ and $N$ into some $M^{\prime}$ in which $\bar{a}^{\wedge} \bar{b}$ and $\bar{c}^{\wedge} \bar{d}$ are identified.

Definition 16. $M$ is said to be $E$-homogeneous, if whenever $\bar{a}, \bar{b}, c \in M$ and $\bar{a} \sim_{M} \bar{b}$, then there is $d \in M$ such that $\bar{a}^{\wedge} c \sim_{M} \bar{b}^{\wedge} d$.

EXAMPLE 17. (i) Let $E$ be defined as follows: for countable $M \vDash T$, and $\bar{a}, \bar{b} \in M$, $\bar{a} \sim{ }_{M} \bar{b}$ iff there is an automorphism $f$ of $M$ such that $f(\bar{a})=\bar{b}$. Then every $M$ is $E$-homogeneous: However $E$ need satisfy neither (I) nor (II).

(ii) Let $E$ be defined by $\bar{a} \sim_{M} \bar{b}$ iff $\operatorname{stp}(\bar{a})=\operatorname{stp}(\bar{b})$. Then $E$ satisfies (II), and the model $M$ is $E$-homogeneous iff it is almost homogeneous. If $T$ is stable and all types have finite multiplicity, then $E$ satisfies $(\mathrm{I})_{M}$ for all $M$.

Proposition 18. Let $M$ and $N$ be countable models, such that $N$ is E-homogeneous $(\mathrm{I})_{N}$ is satisfied, and all types (over $\varnothing$ ) realised in $M$ are realised in $N$. Then $M$ is elementarily embeddable in $N$.

Proof. List $M$ as $\left\{a_{i}: i<\omega\right\}$. I will define $T \subset^{\omega>} \omega$, and $b_{s} \in N$ for $s$ in $T$, inductively, such that

(i) $T$ is closed under subsequences,

and for all $n<\omega$,

(ii) $T(n)=\{s \in T: l(s)=n\}$ is finite,

(iii) for all $s \in T(n), \operatorname{tp}\left(b_{s \uparrow 1}, b_{s \uparrow 2}, \ldots, b_{s}\right)=\operatorname{tp}\left(a_{0}, a_{1}, \ldots, a_{n-1}\right)$,

(iv) whenever $\bar{c}$ is an $n$-tuple from $N$ such that $\operatorname{tp}(\bar{c})=\operatorname{tp}\left(a_{0}, \ldots, a_{n-1}\right)$, then there is some $s \in T(n)$ such that $\bar{c} \sim_{N}\left(b_{s \uparrow 1}, b_{s \uparrow 2}, \ldots, b_{s}\right)$.

Suppose that $T(m)$ and $b_{s}$ for $s \in T(m)$ have been defined for all $m \leqslant n$, satisfying the above conditions. I now define $T(n+1)$ and $b_{s}$ for $s \in T(n+1)$. Let 
$p\left(x_{0}, x_{1}, \ldots, x_{n}\right)$ be $\operatorname{tp}\left(a_{0}, a_{1}, \ldots, a_{n}\right)$. By hypothesis $p$ is realised in $N$. By $(\mathrm{I})_{N}$, for some $r$ with $1 \leqslant r<\omega$, there are $\left\{\bar{c}_{j}: j \leqslant r\right\}$ in $N$, a maximal set of pairwise non- $E_{p}^{N}$-equivalent realisations of $p$ in $N$.

Now consider some $\bar{c}_{j}, j \leqslant r$. So $\bar{c}_{j}$ is $\left(c_{0}^{j}, \ldots, c_{n}^{j}\right)$ say, and clearly $\operatorname{tp}\left(c_{0}^{j}, \ldots, c_{n-1}^{j}\right)$ $=\operatorname{tp}\left(a_{0}, \ldots, a_{n-1}\right)$. Thus by condition (iv) of the induction hypothesis, there is $s \in T(n)$ such that $\left(c_{0}^{j}, \ldots, c_{n-1}^{j}\right) \sim_{N}\left(b_{s \uparrow 1}, \ldots, b_{s}\right)$. Now by the $E$-homogeneity of $N$, there is $b \in N$ such that

$$
\left(c_{0}^{j}, \ldots, c_{n-1}^{j}, c_{n}^{j}\right) \sim_{N}\left(b_{s 11}, \ldots, b_{s}, b\right) .
$$

Let us denote this $s$ and $b$ (which depend on $\bar{c}^{j}$ ) by $s(j)$ and $b^{j}$. For each $s \in T(n)$, let $X_{s}=\{j \leqslant r: s(j)=s\}$, and let $q_{s}=\left|X_{s}\right|$. Put $T(n+1)=\left\{s^{\wedge}\langle i\rangle: s \in T(n)\right.$ and $\left.i<q_{s}\right\}$. We can clearly assign subscripts from $T(n+1)$ to the $b^{j}$ 's, such that for each $s \in T(n)$,

$$
\left\{b^{j}: j \in X_{s}\right\}=\left\{b_{s^{\wedge}\langle i\rangle}: i<q_{s}\right\} .
$$

Thus defining $T$ and $b_{s} \in N$ for $s \in T$, it is clear that conditions (i)-(iv) above are satisfied.

$T$ is clearly infinite, and each element in $T$ has only a finite number of immediate successors. So by König's Lemma, $T$ has an infinite branch, that is, there is $\eta \in{ }^{\omega} \omega$, such that for all $n<\omega, \eta \uparrow n \in T$. By condition (iii),

$$
\operatorname{tp}\left(b_{\eta \mid 1}, \ldots, b_{\eta \mid n}\right)=\operatorname{tp}\left(a_{0}, \ldots, a_{n-1}\right) \text { for all } n<\omega .
$$

Thus the mapping $f$ from $M$ to $N$ defined as $f\left(a_{n}\right)=b_{\eta \uparrow(n+1)}$ for all $n<\omega$, is an elementary map. Thus the proof is complete.

NoTE 19. Consider the following property: (IV) if $\bar{a} \sim_{M} \bar{b}$ then for all $n<l(\bar{a})$, $\bar{a} \mid n \sim{ }_{M} \bar{b} \uparrow n$. Then it is clear that if $E$ is any assignment, then there is an assignment $E^{\prime}$ refining $E$, which satisfies (IV), and such that if $E$ satisfies (I) or (II), then so respectively does $E^{\prime}$, and such that if $M$ is $E$-homogeneous then it is also $E^{\prime}$-homogeneous.

Proposition 20. Let $M$ and $N$ be countable E-homogeneous models, where $E$ satisfies (II), and both $M$ and $N$ satisfy (I). Suppose that $M$ and $N$ realise the same (pure) types. Then $M$ and $N$ are isomorphic.

Proof. First by Proposition 18, we can assume that $M$ is an elementary substructure of $N$. We can also, by Note 19, assume that $E$ satisfies (IV).

We show that for $\bar{a} \in M$ and $\bar{b} \in N$, the relation $\bar{a} \sim_{N} \bar{b}$ is a "back and forth", i.e. for such $\bar{a}$ and $\bar{b}$ with $\bar{a} \sim_{N} \bar{b}$ and $c \in M$ there is $d \in N$ with $\bar{a}^{\wedge} c \sim_{N} \bar{b}^{\wedge} d$, and if again for such $\bar{a}, \bar{b}$, with $\bar{a} \sim_{N} \bar{b}$ and $d \in N$ there is $c \in M$ such that $\bar{a}^{\wedge} c \sim_{N} \bar{b}^{\wedge} d$. This will clearly suffice. The first clause (the "forth") is immediate by the $E$-homogeneity of $N$. So we have to prove now the second clause (the "back"). So let $\bar{a} \in M$ and $\bar{b} \in N$ with $\bar{a} \sim_{N} b$, and let $d \in N$. We seek $c \in M$ with $\bar{a}^{\wedge} c \sim_{N} \bar{b}^{\wedge} d$. Let $p=\operatorname{tp}(\bar{b})(=\operatorname{tp}(\bar{a}))$, and let $q=\operatorname{tp}\left(\bar{b}^{\wedge} d\right)$. Let $\bar{e}_{0}, \bar{e}_{1}, \ldots, \bar{e}_{k-1}(k<\omega)$ be a maximal set of non- $E_{q}^{N}$-equivalent realisations of $q$ in $N$, and we can assume that $\bar{e}_{0}=\bar{b}^{\wedge} d$. Let $\overline{\bar{e}}$ be $\bar{e}_{0}^{\wedge} \ldots \wedge \bar{e}_{k-1}$. Then by assumption, there is $\overline{\bar{e}}^{\prime}$ in $M$ with $\operatorname{tp}(\overline{\bar{e}})=\operatorname{tp}(\overline{\bar{e}})$. So $\overline{\bar{e}}^{\prime}$ is $\bar{e}_{0}^{\prime} \wedge \ldots \wedge \bar{e}_{k-1}^{\prime}$ where the $\bar{e}_{i}^{\prime}$ realise $q$. As $E$ satisfies (II), and 
by Lemma 15 , the $\bar{e}_{i}^{\prime}$ are pairwise non- $E_{q}^{N}$-equivalent. Thus clearly there is $r<k$ such that $\bar{e}_{r}^{\prime} \sim_{N} \bar{e}_{0}$. Let $\bar{a}^{\prime}$ be $e_{r}^{\prime} \uparrow n$ where $n=l\left(\bar{e}_{r}^{\prime}\right)-1$. (So $n=l(\bar{a})=l(\bar{b})$.) Thus, by (IV), $\bar{a}^{\prime} \sim_{N} \bar{b}$. So (by transitivity of $\sim_{N}$ ) $\bar{a}^{\prime} \sim_{N} \bar{a}$, and by (II), $\bar{a}^{\prime} \sim_{M} \bar{a}$. As $M$ is $E$-homogeneous, there is $c \in M$ such that $\bar{e}_{r}^{\prime} \sim_{M} \bar{a}^{\wedge} c$. But $\bar{e}_{r}^{\prime} \sim_{N} \bar{e}_{0}$ and $\bar{e}_{0}$ is $\bar{b}^{\wedge} d$. So by (II) again, we have $\bar{a}^{\wedge} c \sim_{N} \bar{b}^{\wedge} d$, and the proof is complete.

COROLlaRY 21. Suppose that $T$ is $\omega$-stable and all countable models of $T$ are almost homogeneous. Then $I\left(\aleph_{0}, T\right)$ (the number of countable models of $T$, up to isomorphism) is $1, \boldsymbol{\aleph}_{0}$ or $2^{\aleph_{0}}$.

Proof. If $T$ is $\omega$-stable, then $E$ as defined in Example 17(ii) satisfies (II) and (I) $M$ for all $M$. By assumption all countable models of $T$ are $E$-homogeneous, and thus each countable model is determined by the types it realises. It is well known that this implies $I\left(\boldsymbol{\aleph}_{0}, T\right)$ is $1, \boldsymbol{\aleph}_{0}$ or $2^{\aleph_{0}}$.

NoTE 22. (i) Bouscaren and Lascar [1] have shown that for a wide class of $\omega$-stable theories (to be precise, those in which all multidimensional types have infinite dimension), the hypotheses of Corollary 21 are satisfied.

(ii) We cannot omit from Proposition 20, the hypothesis that $E$ satisfies (II). A counterexample is given by the well-known "Ehrenfeucht example", where $E$ is interpreted as in Example 17(i).

\section{REFERENCES}

1. E. Bouscaren and D. Lascar, The countable models of a non-multidimensional w-stable theory (to appear).

2. D. Lascar and B. Poizat, An introduction to forking, J. Symbolic Logic 44 (1979), 330-350.

3. S. Shelah, Classification theory and the number of non-isomorphic models, North-Holland, Amsterdam, 1978.

Department of Mathematics, University of Paris VII, Paris, France

Current address: Department of Mathematics, University of Manchester, Manchester, M13 9PL, England 